\title{
VERZEICHNIS DER MITARBEITERINNEN UND MITARBEITER DIESES BANDES
}

Prof. Dr. Keith Ansell-Pearson, University of Warwick, Department of Philosophy, Coventry CV4 7AL, England, e-mail:<kjap@davidbowie.co.uk >

Prof. Dr. Babette E. Babich, Fordham University, Department of Philosophy, 113 West 60th Street, New York, NY 10023, USA, e-mail:<Babich@mary.fordham.edu >

Martina Bretz, Philosophisches Seminar der Universität Bonn, Lehr- und Forschungsbereich I, Am Hof 1, 53113 Bonn, e-mail:<MartBretz@aol.com>

Dr. Volker Depkat, Universität Greifswald, Historisches Institut, Bahnhofstr. 51, 17487 Greifswald, e-mail:<depkat@mail.uni-greifswald.de>

Prof. Dr. Günter Figal, Philosophisches Seminar der Universität Tübingen, Bursagasse 1, 72070 Tübingen, e-mail:<figal@uni-tuebingen.de>

Univ.-Prof. DDr. Johann Figl, Universität Wien, Kath.-Theol. Fakultät, Institut für Religionswissenschaft, Freyung 6a/2/4/Tür 8, 1010 Wien, Österreich, e-mail: <dagmar.hofko@univie.ac.at>

Prof. Dr. Volker Gerhardt, Humboldt-Universität zu Berlin, Institut für Philosophie, Unter den Linden 6, 10099 Berlin, e-mail:<Volker=Gerhardt@Geschichte.HU-Berlin.de >

Dr. Doris Vera Hofmann, University of Rhode Island, Philosophy Department, 170 Chafee Bldg., Kingston, RI 02881, USA, e-mail:<VTEHofmann@prodigy.net >

Prof. Dr. Boris W. Markow, ul. Richard Sorge 12/84, 198328 Sankt Petersburg/Russia, e-mail:<olga@vi1450.spb.edu>

Prof. Dr. Scarlett Marton, Universidade de Sao Paulo, Departamento de Filosofia, Av. Prof. Luciano Gualberto, 315, BR-05508-900 Sao Paulo, Brasilia, e-mail: <smarton@usp.br >

Prof. Dr. David Owen, Department of Politics/Centre for Post-Analytic Philosophy, University of Southampton, Highfield, Southampton, SO17 1BJ, England, e-mail:<dowen@soton.ac.uk>

Prof. Dr. Robert Pippin, Department of Philosophy, The University of Chicago, 1010 East 59th Street (C/M: CL 17), Chicago, Illinois 60637, USA, e-mail:<r-pippin@uchicago.edu> 
Prof. Dr. Bernard Reginster, Brown University, Department of Philosophy, College Street 54, Box 1918, Providence, RI 02912, USA, e-mail:<Bernard_Reginster@brown.edu>

Prof. Dr. Manfred Riedel, Martin-Luther-Universität Halle-Wittenberg, Institut für Philosophie, 06099 Halle/Saale, e-mail:<riedel@phil.uni-halle.de>

Prof. Dr. Richard Schacht, University of Illinois, Department of Philosophy, 105 Gregory Hall, 810 South Wright Street, Urbana, IL 61801, USA, e-mail:<rschacht@uiuc.edu>

Prof. Dr. Peter Sedgwick, Philosophy Section, Cardiff University, P. O. Box 94, Cardiff CF1 3 XB, England, e-mail:<psedgwick@zdnetonebox.com>

Prof. Dr. Alan D. Schrift, Department of Philosophy, Grinnell College, Grinnell, Iowa 50112-1690, USA, e-mail:<schrift@grinnell.edu>

Prof. Dr. Josef Simon, Birkenweg 29, 53343 Wachtberg-Niederbachem, e-mail:<jg-simon@uni-bonn.de>

Dr. Andreas Urs Sommer, Ernst-Moritz-Arndt-Universität Greifswald, Institut für Philosophie, Baderstr. 6-7, 17487 Greifswald, e-mail:<asommer@mail.uni-greifswald.de>

Prof. Dr. Werner Stegmaier, Ernst-Moritz-Arndt-Universität Greifswald, Institut für Philosophie, Baderstr. 6-7, 17487 Greifswald, e-mail:<stegmai@mail.uni-greifswald.de>

Prof. em. Dr. Helm Stierlin, Kapellenweg 19, 69121 Heidelberg 